\title{
Comparaison de différents systèmes de collecte avec deux types de pièges pour la capture des glossines et des Tabanidés
}

\author{
S. Amsler ${ }^{1}$, J. Filledier ${ }^{1}$
}

AMSLER (S.), FILLEDIER (J.). Comparaison de différents systèmes de collecte avec deux types de pièges pour la capture des glossines et des Tabanidés. Revue Élev. Méd. vét. Pays trop., 1994, 47 (4) : 387-396

Lors d'expériences menées en 1992 et 1994 sur le site de la Comoé, par le Centre international de recherche-développement sur l'élevage en zone subhumide (Bobo-Dioulasso, Burkina Faso), les auteurs ont comparé l'efficacité des bouteilles et des cages associées aux pièges biconique Challier-Laveissière ou monoconique Mérot, comme systèmes de capture pour certaines espèces de glossines (Glossina tachinoides et G. morsitans submorsitans) et les Tabanidae. Pour G. tachinoides, la bouteille ne présente un net intérêt que lorsqu'elle est associée au piège monoconique, mais avec des résultats toujours inférieurs à ceux du piège biconique muni d'une cage. La bouteille avec le piège biconique donne des résultats variables selon les années, mais au mieux équivalents à ceux de la cage. Les différences entre les systèmes sont plus nettes chez les mâles. Pour G. morsitans submorsitans, le système piège biconique/cage reste le plus efficace et la bouteille réduit les captures dans les deux types de pièges. Les captures de Tabanidae sont augmentées (parfois doublées) quand on substitue la bouteille à la cage, quel que soit le type de piège.

Mots-clés : Glossina morsitans submorsitans - Glossina tachinoides Tabanidae - Lutte anti-insecte - Piège - Burkina Faso.

\section{INTRODUCTION}

Depuis quelques années, le piégeage représente un outil efficace de lutte, de prospection, de protection et d'étude pour certaines espèces de glossines. Les pièges peuvent agir de plusieurs façons : en tuant les glossines qui viennent au contact de leur surface (pièges insecticides), en les stérilisant (pièges tunnels) ou en les emprisonnant (pièges de capture) grâce à un système anti-retour. Aussi, l'amélioration des pièges fait-elle l'objet de nombreuses recherches. En effet, les pièges dont on dispose doivent être faciles d'utilisation, et d'un prix abordable, pour permettre la prise en charge durable de la lutte antiglossinienne par les populations locales. Dans cette optique, tous les moyens sont recherchés pour augmenter l'efficacité et baisser le prix de revient de ces techniques : simplification, utilisation de matériaux naturels ou locaux et modification des systèmes de collecte dans le cas des pièges de capture.

Pour évaluer plus facilement l'impact du piégeage, le fait de pouvoir compter les mouches capturées est un atout supplémentaire, d'où l'intérêt des systèmes de collecte placés au sommet du piège. Mais la cage Roubaud clas-

1. CIRDES (anct CRTA), 01 BP 454, Bobo-Dioulasso 01, Burkina Faso.

Reçu le 7.12.1992 (1ère version), accepté le 12.1.1995 (version révisée) sique coûte cher. On a donc comparé son efficacité à celle d'un système constitué de bouteilles de récupération en plastique.

Peu d'études ont été menées en Afrique de l'Ouest sur la modification des systèmes de capture. En République centrafricaine, il semble que la bouteille soit très efficace vis-à-vis de certaines espèces de glossines, lorsqu'elle est utilisée avec un piège bipyramidal. Elle y est même utilisée comme moyen de lutte contre Glossina fuscipes fuscipes. Ce système est deux à trois fois plus efficace et moins cher que le même piège muni d'une cage Roubaud $(3,9,10,11)$. Au Zimbabwe également, ce système de collecte semble donner des résultats intéressants ( $D$. CUISANCE, 1994, comm. pers.). Au Congo, des sacs de plastique à la place des cages ont été utilisés dans la lutte contre Glossina palpalis palpalis lors d'une épizootie de trypanosomose (12). Des expériences antérieures menées au Centre international de recherche-développement sur l'élevage en zone subhumide (CIRDES) avaient donné des résultats encourageants quant à l'efficacité de ce nouveau système (4) vis-à-vis des glossines.

D'autres insectes piqueurs peuvent avoir une importance : les Tabanidae représentent un fléau pour l'élevage par leur nuisance et surtout par leur rôle vecteur dans la transmission mécanique de la trypanosomose animale à Trypanosoma vivax. Le CIRDES a commencé depuis deux ans des recherches sur les systèmes de capture efficaces vis-à-vis de ces insectes $(1,2)$ et la comparaison des bouteilles et des cages fait partie des améliorations envisageables.

\section{MATÉRIEL ET MÉTHODES}

Les expériences sont effectuées en zone tropicale subhumide. Elles se déroulent sur le site du CIRDES, le long de la rivière Comoé, au sud-ouest du Burkina Faso. La densité humaine dans cette région est très faible et la faune sauvage assure la présence de glossines, en particulier de G. m. submorsitans. Une forêt-galerie borde la rivière et ses affluents, le reste de la zone étant constitué d'une savane boisée. Les espèces de glossines rencontrées sont, par ordre décroissant d'importance numérique, Glossina tachinoides Westwood, 1850, G. morsitans submorsitans Newstead, 1910, G. palpalis gambiensis Vanderplank, 1949 et $G$. medicorum Austen, 1911. On trouve également des Tabanidae, principalement du genre Tabanus. 
Deux séries d'expériences sont réalisées pendant deux saisons sèches fraîches (1991-92 et 1993-94). Dans la première série, de janvier à mars 1992, 17 carrés latins $\left(C L^{*}\right) 4 \times 4$ permettent de comparer simultanément 4 pièges sur 4 emplacements, avec rotation aléatoire chaque jour pendant 4 jours. L'efficacité des pièges est alors testée vis-à-vis de G. tachinoides et des Tabanidae. Les pièges comparés sont les suivants :

- piège biconique Challier-Laveissière $(5,6)$, muni d'une cage Roubaud;

- piège biconique équipé du système "bouteille";

- piège monoconique Mérot (7) associé au système "bouteille"; - piège monoconique muni d'une cage Roubaud, témoin.

Le système "bouteille" est représenté dans la photo 1.

Dans la deuxième série d'expériences, de novembre 1993 à janvier 1994, le dispositif est légèrement modifié : le bouchon en plastique de la bouteille est en effet remplacé, dans certains modèles, par du tulle moustiquaire, pour laisser circuler l'air. Le montage est présenté dans la photo 2. Les pièges testés restent les mêmes que dans la première série. On obtient des carrés latins constitués comme suit :

- piège biconique Challier-Laveissière équipé d'une bouteille munie d'un bouchon (= piège biconique /bouteille/bouchon);

- piège biconique/bouteille/moustiquaire ;

- piège monoconique Mérot/bouteille/bouchon ;

- piège monoconique/bouteille/moustiquaire ;

- piège biconique/cage ;

- piège monoconique/cage, témoin.

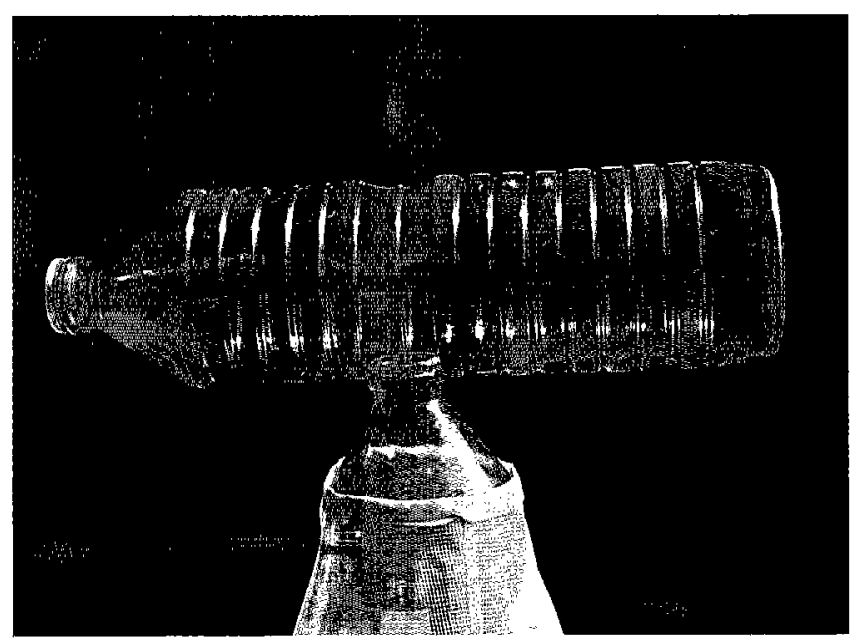

Photo 1: Système de capture composé de deux bouteilles et d'un bouchon plastique.

* ERRATUM : Article paru dans le No1994, 47 (3) : 301-311. Tableau I, lire en légende : $C L$ : carré latin.

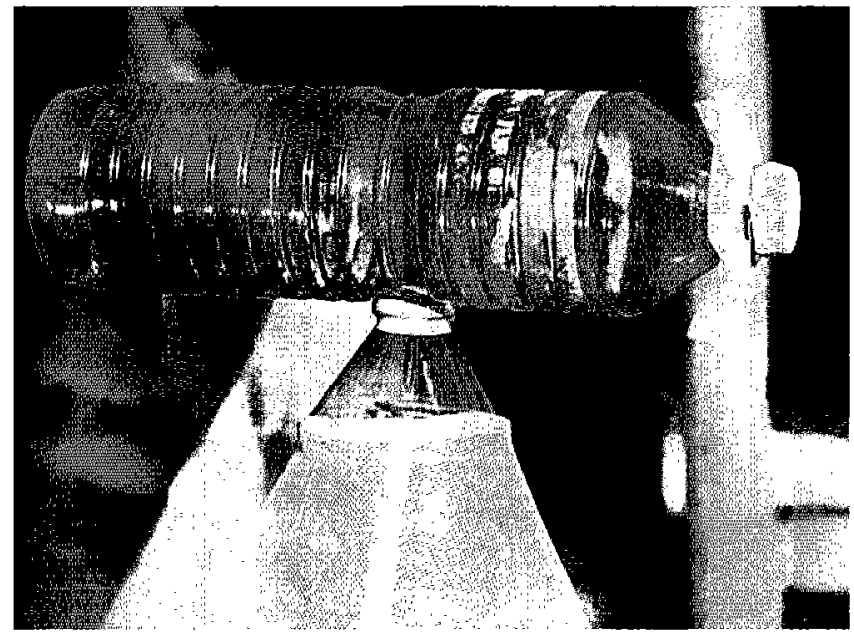

Photo 2 : Système de capture composé de deux bouteilles et d'un tulle moustiquaire.

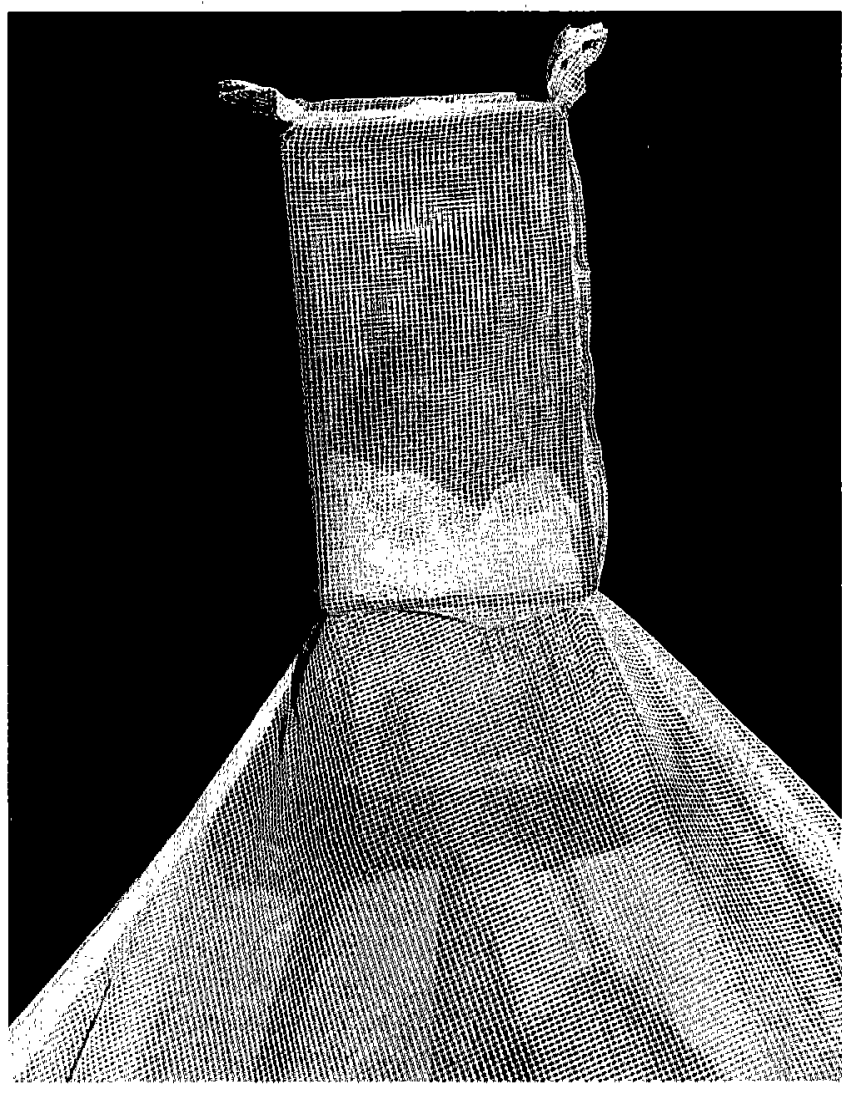

Photo 3 : Système de capture classique composé d'urt montant métallique et d'une cage Roubaud.

Dans ce cas, les études portent sur $G$. tachinoides (12 CL), G. morsitans submorsitans (10 CL) et les Tabanidae $(10 \mathrm{CL})$. Les bouteilles utilisées sont des bouteilles en plastique d'eau minérale, facilement disponibles au Burkina Faso (eau mise en bouteille à Bobo-Dioulasso). La cage de capture est une cage Roubaud classique telle que représentée sur la photo 3 . Les pièges sont confec- 
tionnés au CIRDES. De plus, chaque piège est muni d'un système anti-prédateur (principalement les fourmis) constitué d'une plaque de polystyrène enduite de glu, au centre de laquelle est enfoncé le piquet du piège. Les pièges sont espacés de $500 \mathrm{~m}$ environ.

\section{RÉSULTATS}

Les glossines et les Tabanidés sont récoltés tous les jours à la même heure, selon un circuit fixe, au moment de la rotation des pièges. Pour les glossines, les analyses ne concernent que Glossina tachinoides et $G$. morsitans submorsitans, les autres espèces étant présentes en trop faibles quantités pour aboutir à des conclusions interprétables statistiquement.

Les analyses de variance sont réalisées après transformation logarithmique des données et addition des carrés latins. Les calculs statistiques sont effectués avec un programme CRTA, sur LOTUS 1,2,3 V3. L'index de capture correspond au rapport d'efficacité entre le piège testé et le piège témoin, après correction des variations entre carrés latins. Les résultats sont présentés dans les tableaux $\mathrm{I}$ à $\mathrm{X}$ et les figures 1 et 2.

\section{Glossina tachinoides}

\section{Expérience de 1992 (tableaux I, II)}

Il faut tout d'abord noter que si les quantités de glossines capturées sont généralement faibles, elles sont néanmoins toujours suffisantes pour être statistiquement interprétables. Comme le montrent les tableaux I et II, le piège biconique muni d'une bouteille est le système le moins performant dans les deux sexes, par rapport au même piège équipé d'une cage ou du piège monoconique, alors qu'il est reconnu que le piège biconique classique est plus efficace que le piège monoconique pour cette espèce de glossine. C'est d'ailleurs ce qu'on retrouve dans cette expérience avec les pièges munis de cages. II faut noter cependant que les différences ne sont pas significatives.

On observe également des variations selon le sexe dans l'efficacité des différents systèmes. Chez les mâles, le piège biconique muni d'une bouteille est significativement inférieur au même piège muni d'une cage $(p<0,001)$ et au piège monoconique équipé d'une bouteille $(p<0,01)$. Le système piège biconique/cage est également significativement supérieur ( $p<0,001)$ au système piège monoconique/cage, mais cette différence disparaît quand la bouteille remplace la cage. En revanche, chez les femelles, aucune différence significative n'est notée entre les pièges. La tendance est quand même à la supériorité du piège monoconique sur le piège biconique, quel que soit le système de capture. Cependant, les différences de captures entre les sexes ne sont pas significatives au test du $\chi^{2}$, quel que soit le système employé.

On peut noter dans la figure 1 que les femelles sont collectées en plus grand nombre que les mâles (63 p. 100 de femelles en moyenne dans les récoltes). Le piège biconique associé à la cage donne des quantités capturées équivalentes dans les deux sexes, ce qui semble sortir des normes de récoltes observées ; les femelles devraient en effet être plus nombreuses.

\section{Expérience de 1994}

Le système de collecte a été modifié par changement du bouchon plastique de la bouteille en tulle moustiquaire pour laisser circuler l'air à travers le système. Les tableaux III et IV montrent que le piège biconique représente le meilleur système de capture pour $G$. tachinoides. Quel que soit le système de collecte, cage ou bouteille, il présente des différences significatives vis-à-vis du piège monoconique associé à la cage ( $p<0,05$ à $p<0,01)$ ou à la bouteille avec bouchon ou moustiquaire $(p<0,001)$. En revanche, on ne note pas de différence statistique entre les différents systèmes de collecte associés au piège biconique. Le changement du bouchon par le tulle moustiquaire ne modifie pas statistiquement les résultats des pièges biconique ou monoconique, mais les mâles semblent monter plus facilement que les femelles dans les pièges où la bouteille est équipée d'une moustiquaire.
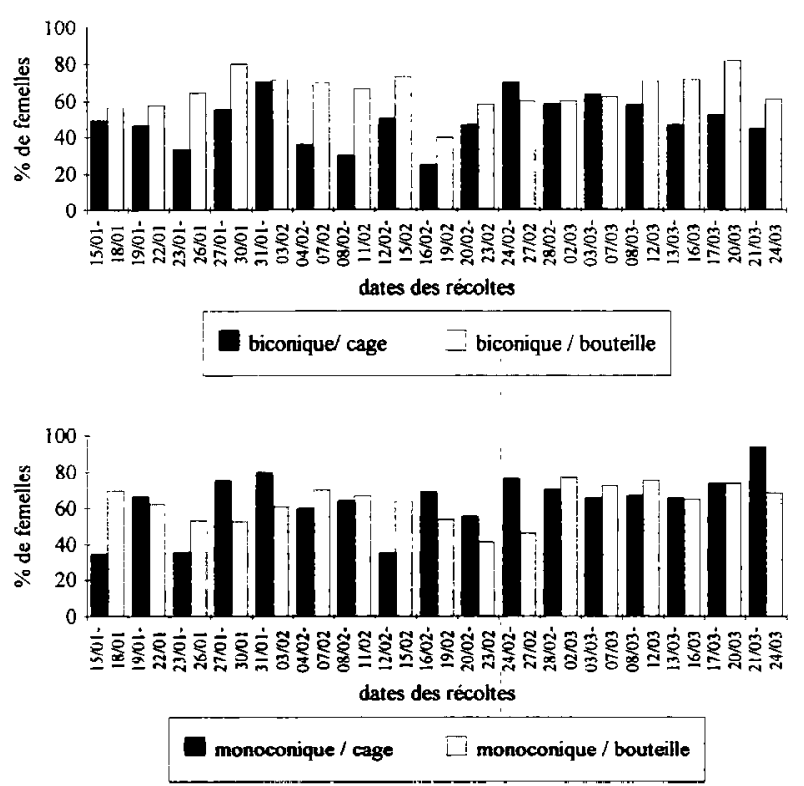

Figure 1 : Evolution du pourcentage de femelles de Glossina tachinoides selon le système de capture et le modèle de piège, du 15.01. au 24.03.1992. 
S. Amsler J. Filledier

TABLEAU I Captures de Glossina tachinoides du 15/01 / 1992 au 24 / 03 / 1992.

\begin{tabular}{|c|c|c|c|c|c|c|c|c|}
\hline $\begin{array}{l}\text { Modèles de } \\
\text { pièges }\end{array}$ & \multicolumn{2}{|c|}{$\begin{array}{l}\text { Piège biconique / } \\
\text { cage }\end{array}$} & \multicolumn{2}{|c|}{$\begin{array}{l}\text { Piège biconique / } \\
\text { bouteille }\end{array}$} & \multicolumn{2}{|c|}{$\begin{array}{l}\text { Piège monoconique / } \\
\text { bouteille }\end{array}$} & \multicolumn{2}{|c|}{$\begin{array}{l}\text { Piège monoconique / } \\
\text { cage ; témoin }\end{array}$} \\
\hline $\mathrm{CL}$ & mâles & femelles & mâles & femelles & mâles & femelles & mâles & femelles \\
\hline $\begin{array}{r}1 \\
2 \\
3 \\
4 \\
5 \\
6 \\
7 \\
8 \\
9 \\
10 \\
11 \\
12 \\
13 \\
14 \\
15 \\
16 \\
17\end{array}$ & $\begin{array}{r}29 \\
35 \\
20 \\
8 \\
5 \\
16 \\
7 \\
8 \\
9 \\
8 \\
3 \\
26 \\
20 \\
5 \\
16 \\
10 \\
10 \\
235\end{array}$ & $\begin{array}{r}28 \\
30 \\
10 \\
10 \\
12 \\
9 \\
3 \\
8 \\
3 \\
7 \\
7 \\
37 \\
35 \\
7 \\
14 \\
11 \\
8 \\
239\end{array}$ & $\begin{array}{r}7 \\
14 \\
5 \\
2 \\
2 \\
3 \\
8 \\
4 \\
3 \\
5 \\
2 \\
4 \\
11 \\
5 \\
10 \\
6 \\
7 \\
98\end{array}$ & $\begin{array}{r}9 \\
19 \\
9 \\
8 \\
5 \\
7 \\
16 \\
11 \\
2 \\
7 \\
3 \\
6 \\
18 \\
12 \\
25 \\
27 \\
11 \\
195\end{array}$ & $\begin{array}{r}16 \\
20 \\
15 \\
9 \\
11 \\
6 \\
4 \\
4 \\
12 \\
10 \\
7 \\
5 \\
12 \\
5 \\
6 \\
9 \\
11 \\
162\end{array}$ & $\begin{array}{r}37 \\
33 \\
17 \\
10 \\
17 \\
14 \\
8 \\
7 \\
14 \\
7 \\
6 \\
17 \\
31 \\
15 \\
11 \\
25 \\
24 \\
293\end{array}$ & $\begin{array}{r}15 \\
7 \\
9 \\
4 \\
3 \\
6 \\
5 \\
11 \\
9 \\
8 \\
6 \\
14 \\
13 \\
5 \\
11 \\
5 \\
1 \\
132\end{array}$ & $\begin{array}{r}8 \\
14 \\
5 \\
12 \\
12 \\
9 \\
9 \\
6 \\
20 \\
10 \\
20 \\
33 \\
25 \\
10 \\
21 \\
14 \\
16 \\
244\end{array}$ \\
\hline Total & \multicolumn{2}{|c|}{474} & \multicolumn{2}{|c|}{293} & \multicolumn{2}{|c|}{455} & \multicolumn{2}{|c|}{376} \\
\hline
\end{tabular}

CL : carré latin.

TABLEAU II Index de capture de Glossina tachinoides du 15 / 01 / 1992 au 24 / 03 / 1992.

\begin{tabular}{|c|c|c|c|c|c|c|}
\hline \multirow{2}{*}{ Pièges } & \multicolumn{2}{|c|}{ Log (moyenne+1) } & \multicolumn{2}{c|}{ Moyenne } & \multicolumn{2}{c|}{ Index de capture } \\
\cline { 2 - 7 } & mâles & femelles & mâles & femelles & mâles & femelles \\
\hline Piège biconique / cage & 0,50 & 0,52 & 2,16 & 2,29 & $1,68^{* * *}$ & $0,944^{\text {Ns }}$ \\
\hline Piège biconique / bouteille & 0,31 & 0,48 & 1,06 & 2,04 & $0,83^{\mathrm{Ns}}$ & $0,84^{\mathrm{Ns}}$ \\
\hline Piège monoconique / bouteille & 0,43 & 0,57 & 170 & 2,74 & $1,33^{\mathrm{Ns}}$ & $1,122^{\mathrm{Ns}}$ \\
\hline Piège monoconique / cage; témoin & 0,36 & 0,54 & 1,28 & 2,43 & 1 & 1 \\
\hline
\end{tabular}

Seuil de signification par rapport au témoin : NS : non significatif ; ${ }^{* * *}: p<0,001$

Là encore, on observe des variations selon le sexe. Comme on le voit sur la figure 2, le piège monoconique semble favoriser les captures de femelles par rapport au piège biconique, ce qui confirme les résultats de la première expérience. De plus, chez les mâles, le piège monoconique équipé d'une cage est significativement moins efficace $(p<0,001)$ que le piège biconique, quel que soit le système de collecte associé à ce dernier, alors qu'on n'observe pas de différence significative chez les femelles. De plus, les seuils de signification des différences de captures entre les différents systèmes sont en général moins élevés chez les femelles.

\section{Glossina morsitans submorsitans}

En 1992, les quantités obtenues sont trop faibles pour être interprétables. Dix carrés latins ont été réalisés du 17.11.1993 au 17.1.1994 avec les systèmes décrits plus haut. Les tableaux $V$ et VI montrent la supériorité significative du piège biconique sur le piège monoconique, quel que soit le système de collecte utilisé; avec cependant des seuils variables. La cage représente le système le plus performant $(p<0,001)$, passant devant la bouteille avec la moustiquaire $(p<0,01$ à $p<0,001)$. Le bouchon donne 
TABLEAU III Captures de Glossina tachinoides du 17 / 11 / 1993 au 29 / 01 / 1994.

\begin{tabular}{|c|c|c|c|c|c|c|c|c|c|c|c|c|}
\hline $\begin{array}{l}\text { Modèles } \\
\text { de } \\
\text { pièges }\end{array}$ & \multicolumn{2}{|c|}{$\begin{array}{c}\text { Piège } \\
\text { biconique / } \\
\text { bouteille / } \\
\text { bouchon }\end{array}$} & \multicolumn{2}{|c|}{$\begin{array}{c}\text { Piège } \\
\text { biconique / } \\
\text { bouteille / } \\
\text { moustiquaire }\end{array}$} & \multicolumn{2}{|c|}{$\begin{array}{c}\text { Piège } \\
\text { monoconique / } \\
\text { bouteille / } \\
\text { bouchon }\end{array}$} & \multicolumn{2}{|c|}{$\begin{array}{c}\text { Piège } \\
\text { monoconique / } \\
\text { bouteille / } \\
\text { moustiquaire }\end{array}$} & \multicolumn{2}{|c|}{$\begin{array}{l}\text { Piège } \\
\text { biconique / } \\
\text { cage }\end{array}$} & \multicolumn{2}{|c|}{$\begin{array}{c}\text { Piège } \\
\text { monoconique / } \\
\text { cage ; témoin }\end{array}$} \\
\hline $\mathrm{CL}$ & mâles & femelles & mâles & femelles & mâles & femelles & mâles & femelles & mâles & femelles & mâles & femelles \\
\hline $\begin{array}{c}1 \\
2 \\
3 \\
4 \\
5 \\
6 \\
7 \\
8 \\
9 \\
10 \\
11 \\
12\end{array}$ & $\begin{array}{c}53 \\
51 \\
32 \\
23 \\
51 \\
31 \\
27 \\
21 \\
15 \\
7 \\
19 \\
19 \\
349\end{array}$ & $\begin{array}{c}124 \\
98 \\
50 \\
56 \\
65 \\
46 \\
13 \\
21 \\
12 \\
11 \\
22 \\
17 \\
535\end{array}$ & $\begin{array}{l}40 \\
25 \\
34 \\
35 \\
20 \\
35 \\
34 \\
12 \\
32 \\
12 \\
29 \\
18 \\
326\end{array}$ & $\begin{array}{c}83 \\
58 \\
68 \\
47 \\
34 \\
47 \\
27 \\
20 \\
17 \\
13 \\
24 \\
9 \\
447\end{array}$ & $\begin{array}{c}21 \\
30 \\
13 \\
10 \\
16 \\
10 \\
12 \\
13 \\
7 \\
11 \\
28 \\
3 \\
174\end{array}$ & $\begin{array}{c}32 \\
72 \\
47 \\
33 \\
40 \\
39 \\
13 \\
25 \\
10 \\
7 \\
22 \\
11 \\
351\end{array}$ & $\begin{array}{c}21 \\
17 \\
10 \\
19 \\
11 \\
12 \\
19 \\
18 \\
9 \\
18 \\
4 \\
8 \\
166\end{array}$ & $\begin{array}{c}45 \\
69 \\
36 \\
43 \\
31 \\
39 \\
20 \\
35 \\
7 \\
17 \\
11 \\
9 \\
362\end{array}$ & $\begin{array}{c}45 \\
70 \\
42 \\
62 \\
45 \\
33 \\
23 \\
29 \\
9 \\
28 \\
22 \\
12 \\
420\end{array}$ & $\begin{array}{c}155 \\
134 \\
80 \\
110 \\
72 \\
42 \\
29 \\
33 \\
26 \\
20 \\
33 \\
7 \\
741\end{array}$ & $\begin{array}{c}22 \\
30 \\
17 \\
18 \\
10 \\
13 \\
16 \\
30 \\
14 \\
14 \\
15 \\
11 \\
210\end{array}$ & $\begin{array}{c}55 \\
51 \\
46 \\
41 \\
18 \\
35 \\
21 \\
26 \\
21 \\
16 \\
32 \\
10 \\
372\end{array}$ \\
\hline Total & \multicolumn{2}{|c|}{884} & \multicolumn{2}{|c|}{773} & \multicolumn{2}{|c|}{525} & \multicolumn{2}{|c|}{528} & \multicolumn{2}{|c|}{1161} & \multicolumn{2}{|c|}{582} \\
\hline
\end{tabular}

CL : Carré latin.

TABLEAU IV Index de capture de Glossina tachinoides du 17/11 / 1993 au 29 / 01 / 1994.

\begin{tabular}{|c|c|c|c|c|c|c|}
\hline \multirow{2}{*}{ Pièges } & \multicolumn{2}{|c|}{ Log (moyenne +1) } & \multicolumn{2}{c|}{ Moyenne } & \multicolumn{2}{c|}{ Index de capture } \\
\cline { 2 - 7 } & mâles & femelles & mâles & femelles & mâles & femelles \\
\hline Piège biconique / bouteille / bouchon & 0,61 & 0,73 & 3,10 & 4,40 & $1,44^{*}$ & $1,24^{\mathrm{Ns}}$ \\
\hline Piège biconique / bouteille / moustiquaire & 0,63 & 0,72 & 3,23 & 4,25 & $1,51^{\star \star}$ & $1,20^{\mathrm{Ns}}$ \\
\hline Piège monoconique / bouteille / bouchon & 0,39 & 0,60 & 1,46 & 3,02 & $0,68^{*}$ & $0,85^{\text {Ns }}$ \\
\hline Piège monoconique / bouteille / moustiquaire & 0,41 & 0,59 & 1,57 & 2,90 & $0,73^{\mathrm{Ns}}$ & 0,82 Ns \\
\hline Piège biconique / cage & 0,66 & 0,81 & 3,61 & 5,53 & $1,68^{* * *}$ & $1,56^{* * *}$ \\
\hline Piège monoconique / cage ; témoin & 0,50 & 0,66 & 2,14 & 3,53 & 1 & 1 \\
\hline
\end{tabular}

Seuil de signification par rapport au témoin : NS : non significatif ; $: p<0,05 ;{ }^{* *}: p<0,01 ; * * *: p<0,001$.

des résultats inférieurs à la moustiquaire, pour les deux types de pièges. Les trois types de pièges biconiques ont par ailleurs des résultats équivalents entre eux.

Dans les deux sexes, la cage est plus efficace, mais dans les pièges munis d'une bouteille, il semble que les femelles préfèrent les bouteilles avec bouchon alors que les mâles entrent de préférence dans les pièges équipés d'une bouteille avec moustiquaire. Chez les mâles, le piège biconique équipé d'une bouteille, avec bouchon ou moustiquaire, donne des seuils de signification variables vis-à-vis du piège monoconique : la différence est hautement significative
( $p<0,001$ ) quand ce dernier est muni d'une bouteille avec bouchon, et les seuils baissent avec la bouteille/moustiquaire $(p<0,01)$ ou la cage $(p<0,05)$. La cage associée au piège biconique augmente les seuils de signification (tabl. IV). Chez les femelles, le piège biconique muni d'une bouteille présente des seuils de signification inférieurs à ceux notés pour les mâles ( $p<0,05$ par rapport au système piège monoconique/cage, $\mathrm{p}<0,05$ à 0,01 par rapport au système monoconique/bouteille/bouchon). On n'observe pas de différence significative avec le piège monoconique équipé d'une bouteille munie d'une moustiquaire. 
TABLEAU V Captures de G. morsitans submorsitans du 17/11/1993 au 17/01/1994.

\begin{tabular}{|c|c|c|c|c|c|c|c|c|c|c|c|c|}
\hline \multirow{2}{*}{$\begin{array}{c}\begin{array}{c}\text { Modèles } \\
\text { de } \\
\text { pièges }\end{array} \\
\mathrm{CL}\end{array}$} & \multicolumn{2}{|c|}{$\begin{array}{c}\text { Piège } \\
\text { biconique / } \\
\text { bouteille / } \\
\text { bouchon }\end{array}$} & \multicolumn{2}{|c|}{$\begin{array}{c}\text { Piège } \\
\text { biconique / } \\
\text { bouteille / } \\
\text { moustiquaire }\end{array}$} & \multicolumn{2}{|c|}{$\begin{array}{c}\text { Piège } \\
\text { monoconique / } \\
\text { bouteille / } \\
\text { bouchon }\end{array}$} & \multicolumn{2}{|c|}{$\begin{array}{c}\text { Piège } \\
\text { monoconique / } \\
\text { bouteille / } \\
\text { moustiquaire }\end{array}$} & \multicolumn{2}{|c|}{$\begin{array}{c}\text { Piège } \\
\text { biconique / } \\
\text { cage }\end{array}$} & \multicolumn{2}{|c|}{$\begin{array}{c}\text { Piège } \\
\text { monoconique / } \\
\text { cage, témoin }\end{array}$} \\
\hline & mâles & femelles & mâles & femelles & mâles & femelles & mâles & femelles & mâles & femelles & mâles & femelles \\
\hline $\begin{array}{r}1 \\
2 \\
3 \\
4 \\
5 \\
6 \\
7 \\
8 \\
9 \\
10\end{array}$ & $\begin{array}{r}11 \\
10 \\
8 \\
5 \\
11 \\
8 \\
7 \\
18 \\
10 \\
13 \\
101\end{array}$ & $\begin{array}{r}15 \\
20 \\
7 \\
13 \\
6 \\
22 \\
12 \\
20 \\
14 \\
33 \\
162\end{array}$ & $\begin{array}{r}8 \\
9 \\
8 \\
25 \\
10 \\
6 \\
9 \\
12 \\
14 \\
11 \\
112\end{array}$ & $\begin{array}{r}16 \\
23 \\
12 \\
32 \\
10 \\
16 \\
7 \\
11 \\
12 \\
16\end{array}$ & $\begin{array}{r}8 \\
5 \\
6 \\
5 \\
4 \\
2 \\
3 \\
10 \\
5 \\
5 \\
53\end{array}$ & $\begin{array}{r}9 \\
9 \\
10 \\
9 \\
7 \\
15 \\
12 \\
9 \\
7 \\
4 \\
91\end{array}$ & $\begin{array}{r}4 \\
5 \\
2 \\
6 \\
7 \\
6 \\
2 \\
13 \\
3 \\
12 \\
60\end{array}$ & $\begin{array}{r}14 \\
11 \\
6 \\
14 \\
10 \\
11 \\
8 \\
13 \\
9 \\
24 \\
120\end{array}$ & $\begin{array}{r}13 \\
7 \\
8 \\
21 \\
9 \\
17 \\
14 \\
13 \\
10 \\
14 \\
126\end{array}$ & $\begin{array}{r}23 \\
19 \\
11 \\
16 \\
14 \\
19 \\
21 \\
24 \\
19 \\
21 \\
187\end{array}$ & $\begin{array}{r}6 \\
4 \\
4 \\
5 \\
7 \\
6 \\
3 \\
14 \\
12 \\
6 \\
67\end{array}$ & $\begin{array}{r}10 \\
18 \\
5 \\
9 \\
6 \\
14 \\
11 \\
13 \\
5 \\
8 \\
99\end{array}$ \\
\hline Total & \multicolumn{2}{|c|}{263} & \multicolumn{2}{|c|}{367} & \multicolumn{2}{|c|}{144} & \multicolumn{2}{|c|}{180} & \multicolumn{2}{|c|}{313} & \multicolumn{2}{|c|}{166} \\
\hline
\end{tabular}

$C L$ : carré latin.
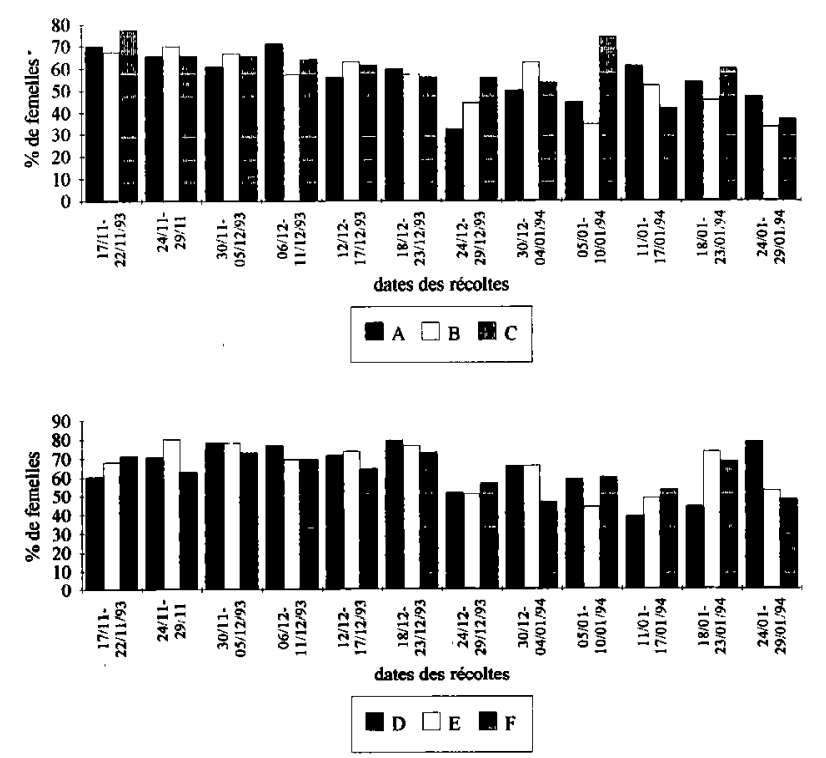

$\begin{array}{ll}\text { A : biconique / bouteille / bouchon } & \mathrm{D}: \text { monoconique / bouteille / bouchon } \\ \mathrm{B}: \text { biconique / bouteille / moustiquaire } & \mathrm{E} \text { : monoconique / bouteille / moustiquaire } \\ \mathrm{C}: \text { biconique : cage } & \mathrm{F}: \text { monoconique / cage / témoin }\end{array}$

Figure 2 : Evolution du pourcentage de femelles de Glossina tachinoides selon le système de capture et le type de piège, du 17.11 .1993 au 29.01.1994

\section{Tabanidae}

\section{Expérience du 15.1.1992 au 24.3.1992}

Dix-sept carrés latins $4 \times 4$ sont réalisés avec les systèmes décrits plus haut (tabl. VII). Le piège monoconique équipé d'une bouteille est significativement supérieur aux autres ( $p<0,001)$, mais les quantités capturées sont faibles et on peut seulement considérer ces données comme unie première tendance. Avec le piège biconique, les captures sont équivalentes quel que soit le système de collecte (tabl. VIII).

\section{Expérience du 17.11.1993 au 17.1.1994}

Dix carrés latins $6 \times 6$ sont effectués pendant cette période et les résultats sont présentés dans les tableaux IX et $X$. Le piège biconique associé à la bouteille, avec bouchon ou moustiquaire, est significativement plus efficace que les pièges, biconique ou monoconique, équipés d'une cage $(p<0,01)$.

Il est à noter également que le piège monoconique associé à la bouteille équipée d'un bouchon capture significativement plus de spécimens que les deux types de pièges avec des cages $(p<0,05)$. On note aussi une différence significative entre le piège biconique équipé d'une bouteille et d'un bouchon et le piège monoconique muni d'une bouteille/moustiquaire, en faveur du premier $(p<0,05)$.

En conclusion, pour les deux types de pièges, il apparaît que les systèmes "bouteille" sont supérieurs au système "cage".

\section{DISCUSSION}

\section{Glossina tachinoides}

Alors que dans la première expérience, le piège biconique équipé d'une bouteille donne des résultats médiocres, on observe en 1994 une efficacité signifiçativement supérieure de ce système sur le piège monoco- 
TABLEAU VI Index de capture de G. morsitans submorsitans du 17/11/1993 au 29 / 01 / 1994.

\begin{tabular}{|c|c|c|c|c|c|c|}
\hline \multirow{2}{*}{ Pièges } & \multicolumn{2}{|c|}{ Log (moyenne +1) } & \multicolumn{2}{|c|}{ Moyenne } & \multicolumn{2}{c|}{ Index de capture } \\
\cline { 2 - 6 } & mâles & femelles & mâles & femelles & mâles & femelles \\
\hline Piège biconique / bouteille / bouchon & 0,36 & 0,48 & 1,28 & 2,02 & $1,47^{*}$ & $1,54^{*}$ \\
\hline Piège biconique / bouteille / moustiquaire & 0,38 & 0,46 & 1,39 & 1,91 & $1,60^{\star}$ & $1,46^{*}$ \\
\hline Piège monoconique / bouteille / bouchon & 0,21 & 0,35 & 0,63 & 1,24 & $0,73^{\mathrm{Ns}}$ & 0,95 Ns \\
\hline Piège monoconique / bouteille / moustiquaire & 0,24 & 0,38 & 0,73 & 1,42 & $0,84^{\mathrm{Ns}}$ & $1,08^{\mathrm{Ns}}$ \\
\hline Piège biconique / cage & 0,41 & 0,55 & 1,60 & 2,58 & $1,84^{\star *}$ & $1,98^{\star * *}$ \\
\hline Piège monoconique / cage ; témoin & 0,27 & 0,36 & 0,87 & 1,31 & 1 & 1 \\
\hline
\end{tabular}

Seuil de signification par rapport au témoin: NS : non significatif ; ${ }^{\star}: p<0,05 ;{ }^{\star \star}: p<0,01 ;{ }^{\wedge+1}: p<0,001$.

TABLEAU VII Captures de Tabanidés du 15/01/1992 au 24/ $03 / 1992$.

\begin{tabular}{|c|c|c|c|c|}
\hline Modèles & $\begin{array}{c}\text { Piège } \\
\text { biconique / } \\
\text { cage }\end{array}$ & $\begin{array}{c}\text { Piège } \\
\text { biconique / } \\
\text { bouteille }\end{array}$ & $\begin{array}{c}\text { Piège } \\
\text { monoconique } / \\
\text { bouteille }\end{array}$ & $\begin{array}{c}\text { Piège } \\
\text { monoconique / } \\
\text { cage ; témoin }\end{array}$ \\
\hline $\begin{array}{r}1 \\
2 \\
3 \\
4 \\
5 \\
6 \\
7 \\
8 \\
9 \\
10 \\
11 \\
12 \\
13 \\
14 \\
15 \\
16 \\
17\end{array}$ & $\begin{array}{r}29 \\
14 \\
4 \\
11 \\
4 \\
5 \\
9 \\
5 \\
3 \\
1 \\
2 \\
6 \\
2 \\
0 \\
3 \\
1 \\
2\end{array}$ & $\begin{array}{r}22 \\
18 \\
8 \\
2 \\
4 \\
8 \\
8 \\
6 \\
2 \\
2 \\
1 \\
2 \\
1 \\
2 \\
1 \\
2 \\
4\end{array}$ & $\begin{array}{r}26 \\
12 \\
4 \\
9 \\
5 \\
20 \\
21 \\
3 \\
10 \\
15 \\
10 \\
14 \\
2 \\
7 \\
5 \\
8 \\
1\end{array}$ & $\begin{array}{r}10 \\
10 \\
0 \\
2 \\
5 \\
10 \\
9 \\
2 \\
4 \\
1 \\
2 \\
4 \\
2 \\
0 \\
4 \\
0 \\
1\end{array}$ \\
\hline Total & 101 & 93 & 172 & 66 \\
\hline
\end{tabular}

nique, quel que soit le système de collecte associé à ce piège. Les chiffres étant plus conséquents dans la deuxième expérience, les résultats sont vraisemblablement plus réalistes. Le piège biconique, avec une cage ou une bouteille, reste le système le plus performant pour capturer cette espèce de glossine.

Le changement du bouchon par le tulle moustiquaire ne modifie pas les résultats des captures, ce qui tend à infirmer la thèse de l'effet de serre suggérée pour expliquer la moindre efficacité du système piège/bouteille par rapport au même piège/cage. Si l'on veut utiliser le bouchon,
TABLEAU VIII Index de capture des Tabanidés du 15 / 01 / 1992 au $24 / 03 / 1992$.

\begin{tabular}{|c|c|c|c|}
\hline $\begin{array}{c}\text { Type } \\
\text { de piège }\end{array}$ & $\begin{array}{c}\text { Log } \\
\text { (moyenne +1) }\end{array}$ & Moyenne & $\begin{array}{c}\text { Index } \\
\text { de capture }\end{array}$ \\
\hline $\begin{array}{c}\text { Piège biconique / } \\
\text { cage }\end{array}$ & 0,28 & 0,89 & $1,43^{\mathrm{Ns}}$ \\
$\begin{array}{c}\text { Piège biconique / } \\
\text { bouteille }\end{array}$ & 0,26 & 0,83 & $1,33^{\mathrm{Ns}}$ \\
$\begin{array}{c}\text { Piège monoconique / } \\
\text { bouteille }\end{array}$ & 0,42 & 1,63 & $2,63^{\star \star \star}$ \\
$\begin{array}{c}\text { Piège monoconique / } \\
\text { cage ; témoin }\end{array}$ & 0,21 & 0,62 & 1 \\
\hline
\end{tabular}

Seuil de signification par rapport au témoin : NS : non significatif ; ${ }^{* * *}: p<0,001$.

il n'est donc pas besoin de modifier son aspect original par du tulle moustiquaire, ce qui augmenterait son coût de fabrication.

Dans le cas du piège monoconique, la bouteille, munie d'un bouchon ou d'un tulle moustiquaire, donne des résultats supérieurs ou au moins équivalents à la cage, mais toujours inférieurs à ceux du piège biconique. La bouteille donne des résultats équivalents à la cage et peut donc remplacer cette dernière sans perte d'efficacité.

Une expérience préliminaire a été menée en 1991 (MEROT, FILLEDIER, comm. pers.). L'analyse des résultats semblait montrer une supériorité des pièges, biconique ou monoconique, équipés d'une bouteille, mais déjà la bouteille paraissait plus intéressante pour le piège monoconique.

Les variations observées d'une année à l'autre sont difficilement explicables. La saison y joue sans doute un rôle important mais délicat à apprécier. De plus, contrairement à d'autres pays d'Afrique, ce système se révèle ici peu efficace vis-à-vis d'une espèce du sous-genre Nemorhina. 
TABLEAU IX Captures de Tabanidés du 17/11/1993 au 17/01/1994.

\begin{tabular}{|c|c|c|c|c|c|c|}
\hline $\begin{array}{l}\text { Modèles de } \\
\text { pièges }\end{array}$ & $\begin{array}{c}\text { Piège } \\
\text { biconique / } \\
\text { bouteille / } \\
\text { bouchon }\end{array}$ & $\begin{array}{c}\text { Piège } \\
\text { biconique / } \\
\text { bouteille / } \\
\text { moustiquaire }\end{array}$ & $\begin{array}{c}\text { Piège } \\
\text { monoconique / } \\
\text { bouteille / } \\
\text { bouchon }\end{array}$ & $\begin{array}{c}\text { Piège } \\
\text { monoconique / } \\
\text { bouteille / } \\
\text { moustiquaire }\end{array}$ & $\begin{array}{c}\text { Piège } \\
\text { biconique / } \\
\text { cage }\end{array}$ & $\begin{array}{c}\text { Plège } \\
\text { monoconique / } \\
\text { cage ; témoin }\end{array}$ \\
\hline $\begin{array}{r}1 \\
2 \\
3 \\
4 \\
5 \\
6 \\
7 \\
8 \\
9 \\
10\end{array}$ & $\begin{array}{r}6 \\
2 \\
11 \\
19 \\
13 \\
29 \\
24 \\
17 \\
34 \\
8\end{array}$ & $\begin{array}{r}13 \\
9 \\
13 \\
14 \\
18 \\
19 \\
15 \\
11 \\
30 \\
14\end{array}$ & $\begin{array}{r}3 \\
6 \\
11 \\
14 \\
10 \\
32 \\
15 \\
13 \\
16 \\
19\end{array}$ & $\begin{array}{r}4 \\
5 \\
8 \\
14 \\
16 \\
12 \\
13 \\
8 \\
22 \\
8\end{array}$ & $\begin{array}{r}6 \\
3 \\
4 \\
21 \\
18 \\
10 \\
15 \\
9 \\
5 \\
11\end{array}$ & $\begin{array}{r}2 \\
7 \\
7 \\
12 \\
3 \\
14 \\
14 \\
13 \\
10 \\
8\end{array}$ \\
\hline Total & 163 & 156 & 139 & 110 & 102 & 90 \\
\hline
\end{tabular}

$C L$ : carré latin.

TABLEAU X Index de capture des Tabanidés du 17/11/1993 au 17 / 01 / 1994.

\begin{tabular}{|c|c|c|c|}
\hline Modèles de pièges & $\begin{array}{c}\text { Log } \\
\text { (moyenne + 1) }\end{array}$ & Moyenne & $\begin{array}{c}\text { Index de } \\
\text { capture }\end{array}$ \\
\hline $\begin{array}{c}\text { Piège biconique / } \\
\text { bouteille / bouchon }\end{array}$ & 0,46 & 1,89 & $1,89^{* *}$ \\
\hline $\begin{array}{c}\text { Piège biconique / bouteille / } \\
\text { moustiquaire }\end{array}$ & 0,44 & 1,73 & $1,73^{\text {** }}$ \\
\hline $\begin{array}{c}\text { Piège monoconique / } \\
\text { bouteille / bouchon }\end{array}$ & 0,41 & 1,59 & $1,59^{*}$ \\
\hline $\begin{array}{c}\text { Piège monoconique / } \\
\text { bouteille / moustiquaire }\end{array}$ & 0,35 & 1,23 & $1,23^{\mathrm{Ns}}$ \\
\hline $\begin{array}{c}\text { Piège biconique / cage } \\
\text { Piège monoconique / } \\
\text { cage témoin }\end{array}$ & 0,30 & 1,02 & $1,02^{\mathrm{NS}}$ \\
\hline
\end{tabular}

Seuil de signification par rapport au témoin : NS : non significatif ; ${ }^{*} p<0,05$; ${ }_{* *}: p<0,01$.

On note des variations dans l'efficacité des différents systèmes selon le sexe : il n'y a jamais de différence significative chez les femelles avec la bouteille, alors qu'on les observe chez les mâles (deuxième expérience). On retrouve également dans tous les cas une majorité de femelles dans les captures. La bouteille ne modifie donc pas la sexratio rencontrée lors des sondages entomologiques.

\section{Glossina morsitans submorsitans}

Le système piège biconique/cage est supérieur aux autres modèles. II n'existe pas d'étude sur l'intérêt de la bouteille pour la capture des glossines de savane en Afrique de l'Ouest, mais il ressort de nos résultats que le piège biconique est toujours significativement supérieur au piège monoconique et que la bouteille réduit les captures pour les deux types de pièges. Il est vrai cependant que les pièges testés dans nos expériences no représentent pas les pièges les plus efficaces sur cette sousespèce de glossine.

\section{Tabanidae}

C'est la première étude concernant la modification du système de collecte pour les Tabanidés. Pour ces vecteurs, la bouteille augmente de façon significative les captures, quel que soit le type de piège. Cependant, le changement du bouchon par du tulle moustiquaire ne modifie pas les résultats. On peut donc choisir la solution la plus simple et la moins coûteuse, à savoir le bouchon d'origine.

Selon la période, les captures les plus importantes sont notées avec le piège biconique ou monoconique. Peut-être doit-on y voir une variation dans les populations de Tabanidés, avec l'émergence dans la deuxième partie de la saison sèche fraîche d'espèces qui répondent mieux au piège biconique. Comme les glossines, ces insectes ont des comportements variables selon les espèces et la période de l'année $(8,13)$. Une étude approfondie de la faune des Tabanidae est souhaitable pour affiner ces premiers résultats. 


\section{CONCLUSION}

Dans des expériences menées dans d'autres pays (9), sur des glossines du sous-genre Nemorhina, la cage Roubaud montre l'inefficacité de son système antiretour, alors que le système "bouteille", identique à celui utilisé dans les expériences présentes, donne des résultats 1,5 à 2 fois supérieurs. Ce système permet également de tuer les glossines plus rapidement par le seul effet de la chaleur.

Mais, ici, on ne retrouve jamais cette supériorité nette de la bouteille sur la cage (au mieux les captures sont équivalentes), en raison peut-être des pièges différents qui sont utilisés et donc du comportement différent des glossines vis-à-vis de ces systèmes. Ces modèles étant cependant utilisés sur le terrain au Burkina Faso et les sondages entomologiques concernant les glossines riveraines, les systèmes de collecte doivent également être adaptés.

Dans le cadre de la lutte conjointe contre les glossines riveraines et les Tabanidés, l'utilisation du piège monoconique muni d'une bouteille en plastique est envisageable et permet d'obtenir des résultats satisfaisants pour un coût acceptable. L'imprégnation de l'intérieur de la bouteille par un insecticide (deltaméthrine par exemple), comme cela est déjà pratiqué dans d'autres pays, permet surtout d'éviter la prédation, par les fourmis principalement (10). De plus, le remplacement de la bouteille lorsqu'elle est abîmée ne pose pas de problème dans les pays où les bouteilles d'eau minérale vides se trouvent facilement sur le marché. II faut cependant remarquer que la bouteille en plastique a tendance à se dégrader assez rapidement et doit être renouvelée fréquemment, ce qui peut poser problème dans de larges campagnes de lutte.

Pour des sondages entomologiques cependant, où l'objectif est de capturer le maximum de spécimens, il semble plus judicieux de favoriser le piège biconique Challier-Laveissière équipé d'une cage.

\section{REMERCIEMENTS}

Ce travail a été réalisé grâce au financement français du Fonds d'Aide et de Coopération (FAC). Nous tenons également à remercier le $\mathrm{Dr}$ S.M. TOURE, directeur du CIRDES, et l'équipe de la Comoé pour la réalisation des expériences.

\section{BIBLIOGRAPHIE}

1. AMSLER (S.), FILLEDIER (J.). Attractivité pour les Tabanidae de l'association méta-crésol/octénol. Résultats obtenus au Burkina Faso. Revue Élev. Méd. vét. Pays trop., 1994, 47 (1) : 93-96.

2. AMSLER (S.), FILLEDIER (J.), MILLOGO (R.). Attractivité pour les Tabanidae de différents pièges à glossines, avec ou sans attractifs olfactifs Résultats préliminaires obtenus au Burkina Faso. Revue Élev. Méd. vét. Pays trop., 1994, 47 (1) : 63-68

3. BLANC (F.), GOUTEUX (J.P.), CUISANCE (D.), POUNEKROZOU (E.), LE MASSON (A.), N'DOKOUÉ (F.), MAINGUET (M.), D'AMICO (F.), LE GALL (F.). La lutte par piégeage contre Glossina fuscipes fuscipes pour la protection de l'élevage en République centrafricaine. III. Vulgarisation en milieu M'Bororo. Revue Élev. Méd. vét. Pays trop., 1991, 44 (3) : 301-307

4. Centre de Recherches sur les Trypanosomoses animales. Rapport annucl. 1991.Bobo Dioulasso, Burkina Faso, CRTA. 45 p.

5. CHALLIER (A.), EYRAUD (M.), LAFAYE (A.), LAVEISSIÈRE (C.). Amélioration du rendement du pic̀ge biconique pour glossines (Diptera : Glossinidae) par l'emploi d'un cône inférieur bleu. Cah. ORSTOM, Sér. Ent. méd. Parasit., 1977, 15 : 283-286.

6. CHALLIER (A.), LAVEISSIÈRE (C.). Un nouveau piège pour la capture des glossines (Glossina : Diptera, Muscidae). Description et essais sur le terrain. Cah. ORSTOM, Sér. Ent. méd. Parasii., 1973, 11 : 251-262.

7. CUISANCE (D.). Le piégeage des tsé-tsé. Maisons-Alfort, IEMVT, 1989. 172 p. (Etudes et Synthèses n ${ }^{\circ} 32$ )

8. DJITEYE (A.). Aperçu sur l'efficacité comparative de différents pièges et odeurs contre les mouches piqueuses (Diptera : Tabanidae et Muscidae) d'intérêt vétérinaire au Mali. In : Premier séminaire international sur les trypanosomoses animales non transmises par les glossines, Annecy, France, 14-16 octobre 1992. $5 \mathrm{p}$.

9. GOUTEUX (J.P.), CUISANCE (D.), DEMBA (D.), N'DOKOUÉ (F.), LE GALL (F.). La lutte par piégeage contre Glossina fuscipes fuscipes pour la protection de l'élevage en République centrafricaine. I. Mise au point d'un piège adapté à un milieu d'éleveurs semi-nomades. Revue Élev. Méd. vét. Pavs trop., 1991, 44 (3) : 287-294.

10. GOUTEUX (J.P.), LANCIEN (C.). Le piège pyramidal à tsé-tsé (Diptera : Glossinidae) pour la capture et la lutte. Essais comparatifs et description de nouveaux systèmes de capture. Trop. Med. Parasit., 1986, $37(1): 61-66$

11. GOUTEUX (J.P.), LE GALL (F.). Piège pyramidal à tsé-tsé pour la protection de l'élevage en République centrafricaine. Revue mond. Zootech. $1992,70 / 71(1-2): 37-43$

12. NOIREAU (F.). OKAMBA-OSSEKE (F.). GOUTEUX (J.P.). Impact immédiat d'une lutte antivectorielle par piégeage sur I'épizootie de trypanosomose au Sud-Congo. Revue Élev. Méd. vét. Pays trop., 1990, 43 (1) : $93-96$.

13. OVAZZA (M.), RICKENBACH (A.), VALADE (M.). Tabanidés de la région de Bobo-Dioulasso (Haute-Volta). Répartition et rythme annuel ; quelques notes de systématique. Bull. Soc. Path. exot., 1959, 52 (5) : 679-698 


\section{S. Amsler J. Filledier}

AMSLER (S.), FILLEDIER (J). Comparison of different systems for collecting tsetse and horse flies with two types of trap. Revue Elev. Méd. vét. Pays trop., 1994, 47 (4): 387-396

The efficiency of biconical (Challier-Laveissière) and monoconical (Mérot) traps with a cage or a bottle as the collecting system for Glossina tachinoides, G. morsitans submorsitans and the Tahanidae was evaluated in 1992 and 1994 at the Comoé site, Burkina Faso by CIRDES. The bottle increased the catches of $G$. tachinoides only when used with the monoconical trap, but the numbers collected were always smaller than with the biconical trap/cage system. The biconical trap with a bottle gave results which varied according to the year concerned, but these results were never more than equal to those with the cage. The differences were more significant in the catches of males. The biconical trap associated with a cage was the most efficient system for catching Glossina morsitans submorsitans. The bottle reduced the catches with both types of trap. The use of bottle increased (and sometimes doubled) the catches of horse flies with both types of trap.

Key-words: Glossina morsitans submorsitans - Glossina tachinoides Tabanidae - Insect control - Trap - Burkina Faso.
AMSLER (S.), FILLEDIER (J). Comparación de diferentes sistemas de colecta mediante dos tipos de trampas para la captura de glosinas y tabánidos. Revue Élev. Méd. vét. Pays trop., 1994, 47 (4) : 387-396

En 1992 y 1994, a lo largo del río Comoe, el Centro Internacional de Investigación-Desarrollo sobre la Crianza en zona sub-húmeda (CIRDES, Bobo-Dioulasso, Burkina Faso), llevó a cabo experiencias, mediante las cuales los autores compararon la eficiencia de botellas y jaulas, en asociación con trampas bicónica de Challier-Laveissière o monocónica de Mérot, como sistemas de captura para ciertas especies de glosinas (Glossina tachinoides y $G$. morsitans submorsitans) y Tabanidae. Para la Glossina tachinoides, la botella representa un interés, unicamente en asociación con la trampa monocónica, pero los resultados son inferiores que los de la trampa bicónica con jaula. Con la botella y la trampa bicónica los resultados varían según el año, pero son, en el mejor de los casos, equivalentes a los de la jaula. Las diferencias entre los sistemas son más nítidas con los machos. En el caso de $G$. morsitans submorsitans, el sistema de trampa bicónica/jaula es el más eficiente y se observa una reducción de las capturas con la asociación de la botella y dos tipos de trampas. Con cualquier tipo de trampa, las capturas de Tabanidae son superiores ( $y$ algunas veces se duplican) cuando la jaula sustituye a la botella.

Palabras clave : Glossina morsitans submorsitans - Glossina tachinoides - Tabanidae - Control de insectos - Trampa - Burkina Faso. 